\title{
Mariage : Essai de synthèse sur les rituels du mariage berbère
}

\section{Abrous}

\section{(2) OpenEdition}

1 Journals

Édition électronique

URL : http://journals.openedition.org/encyclopedieberbere/473

DOI : $10.4000 /$ encyclopedieberbere.473

ISSN : 2262-7197

Éditeur

Peeters Publishers

\section{Édition imprimée}

Date de publication : 29 décembre 2010

Pagination : 4619-4626

ISBN : 978-90-429-2367-6

ISSN : 1015-7344

\section{Référence électronique}

D. Abrous, « Mariage : Essai de synthèse sur les rituels du mariage berbère », Encyclopédie berbère [En ligne], 30 | 2010, document M41c, mis en ligne le 22 septembre 2020, consulté le 15 octobre 2020. URL : http://journals.openedition.org/encyclopedieberbere/473 ; DOI : https://doi.org/10.4000/ encyclopedieberbere.473

Ce document a été généré automatiquement le 15 octobre 2020.

(c) Tous droits réservés 


\title{
Mariage : Essai de synthèse sur les rituels du mariage berbère
}

\author{
D. Abrous
}

1 Le témoignage précédent relatif au mariage transmis par Fadhma Aït-Mansour à sa fille Marguerite Taos Amrouche traite plus précisément des noces. Quelques détails (origine des musiciens : Isahliwen; nom d'une épice ifelfel azebbas) indiquent qu'il s'agit des noces chez les Aït-Abbas, tribu d'origine de la famille Amrouche. Ce texte retrace les étapes principales qui, sur une durée de trois jours, jalonnent les cérémonies du mariage en Kabylie; y sont mentionnés des pratiques et des rites comme : la préparation du repas des noces, l'imposition du henné, la toilette de la mariée, la constitution du cortège nuptial, les cadeaux offerts aux parents de la mariée, les différentes réjouissances.

2 Dans ses grandes lignes, ce texte rejoint la description des cérémonies de mariage faite par Germaine Laoust-Chantréaux aux Aït-Hichem (Kabylie) pour la même période (1937-1939). Jusqu'à un passé relativement récent, c'est-à-dire jusqu'au milieu du XX siècle, la célébration du mariage donnait lieu en Kabylie à un ensemble de manifestations rituelles riche et cohérent qui comportait entre autres, des joutes poétiques, une épreuve de tir à la cible et, dans certaines régions, une mascarade* (Abrous 1992).

3 Le rapport de cohérence qui lie toutes ces manifestations, c'est-à-dire la logique qui les sous-tend, apparaît clairement lorsque l'on tente un rapprochement entre les données kabyles et le matériau ethnographique très riche relatif aux noces collecté par Emile Laoust dès 1915 dans le Moyen-Atlas et le Haut-Atlas (Laoust 1993).

4 Ces manifestations rituelles de très haute teneur symbolique sont concentrées au moment des noces; ce moment est capital car :

- L'alliance scellée entre les deux familles après de longues et discrètes démarches est rendue publique, d'où les formes très diversifiées que revêt la « compétition d'honneur » entre ces deux familles.

- La fiancée quitte sa famille d'origine pour rejoindre celle de son futur époux ; ce moment de passage, perçu comme un moment d'extrême vulnérabilité, est accompagné d'un déploiement de rites aussi bien prophylactiques que propitiatoires. 
Le terme qui désigne les noces : tameyra, plur. timeyriwin, est attesté en kabyle, en tamazight et en tachelhit, et quasiment dans tout le monde berbère Nord ${ }^{1}$. Dans une société où l'honneur est une référence cardinale, les deux familles qui viennent de sceller une alliance se livrent, au moment de la célébration du mariage, à une véritable " compétition d'honneur", compétition destinée non pas à tester (les tests sont faits lors de démarches antérieures, plus discrètes) mais à démontrer avec éclat l'égalité en honneur des deux familles alliées. (cf. Abrous 1992). Cette " compétition d'honneur ", réglée sur « la dialectique du défi et de la riposte » (Bourdieu 1972), se déploie dans les moments les plus marquants de ces cérémonies; elle est perceptible dans :

- Le subtil ordonnancement des joutes poétiques même en l'absence des anciennes joutes savamment codées (Abrous 1992), l'Appel à la Joie tel que noté par Marguerite Taos Amrouche ; $a$ win umi ferhey yas-dyerr-iyi, encore très vivant en Kabylie, est un rappel du principe de réciprocité dans les échanges.

- L'épreuve de tir à la cible et les démonstrations guerrières. Le tir à la cible est un défi imposé par les parents de la mariée; cette épreuve se déroulait en même temps que la joute poétique au cours de laquelle les femmes rivalisaient d'éloquence, la tradition kabyle associant étroitement : awal d wuzzal, « le Verbe et le Fer » (= l'adresse dans le maniement des armes). Cette épreuve de tir à la cible existe dans le Moyen-Atlas; la cible, ici, est un roseau ou un bâton que porte la fiancée au moment où elle quitte la maison paternelle (Laoust 1993, p. 60 et 73). Enfin, la forme la plus courante de ces démonstrations guerrières est la présence du fusil, symbole de l'honneur par excellence. Comme en Kabylie, les fusils «parlent » dans la quasi-totalité des textes collectés par E. Laoust; ils deviennent particulièrement « éloquents» au moment de «l'enlèvement » de la mariée car le passage de la mariée de la maison paternelle vers celle de son futur époux prend la forme d'un véritable enlèvement ${ }^{2}$.

- «L'enlèvement » de la mariée. Evoquant le mariage comme un moment de passage pour la fiancée de la maison, donc de l'autorité, de son père vers celle de son futur époux, Germaine Laoust-Chantréaux affirme qu'il comporte « des rites de séparation et des rites d'agrégation avec, dans l'intervalle, un enlèvement ». (Laoust-Chantréaux 1990, p. 189). Cet aspect a été analysé pour la Kabylie en relation avec les joutes poétiques: c'est cette conquête de la mariée par le Verbe qui explique l'expression de « rapt symbolique » (Abrous 1992). Le texte de Marguerite Taos Amrouche décrit le faste du cortège nuptial dans lequel « Les hommes défilent dans leurs belles draperies blanches, fusils sur l'épaule... » et apporte un détail supplémentaire: la fiancée ne se trouve pas dans la maison paternelle, "elle s'est discrètement éloignée de chez elle pour aller se cacher dans une maison amie, comme le demande la coutume »: l'inaccessibilité de la mariée, très clairement signifiée dans les joutes, est, ici, effective. Sur ce point précis, les données kabyles rejoignent les données collectées par E. Laoust dans le Moyen-Atlas et le Haut-Atlas où d'imposantes démonstrations guerrières accompagnent «l'enlèvement» de la mariée, se poursuivant quelques fois dans le domicile de l'époux. (Laoust 1993, p. 96, 114, 144...). Chez les Imeghran, la porte de l'ighrem * dans lequel se trouve la fiancée est fermée devant le cortège nuptial ; s'engage, alors, pour l'ouvrir une rude bataille rituelle (Laoust 1993, p. 140, 141).

6 Les démonstrations guerrières des «noces berbères " relèvent d'une mise en scène aussi savamment codée que celle des joutes poétiques : pour que l'égalité en honneur entre les deux familles alliées revête tout son éclat en ce jour solennel, il faut que la mariée, inaccessible, soit conquise de haute lutte par le Verbe et par les Armes, d'où "l'éloquence» des fusils. Ce lien entre honneur, guerre et alliance est également présent dans faire touarègue (cf. Claudot-Hawad et Hawad 1993, p. 25 et 26). 
7 - Les dépenses ostentatoires : ces dépenses relèvent, elles aussi, de la "compétition d'honneur "'. On note partout, tel que le décrit le texte de Taos Amrouche, le faste des festins servis à l'occasion des noces; c'est dans la même logique qu'est exposé le trousseau de la mariée (voir le texte de Taos Amrouche, Laoust-Chantréaux 1990, p. 200, et Laoust 1993 ; p. 151)

8 En règle générale, les cérémonies de mariage exigent des dépenses telles que certaines familles sont contraintes de contracter de lourdes dettes; ces dépenses qui défient toute rationalité économique mettent en valeur «le capital symbolique». Cet aspect ostentatoire est perceptible, enfin, dans les échanges de cadeaux entre les deux familles alliées; ces cadeaux sont toujours portés " ostensiblement » comme l'écrit E. Laoust (1993, p. 72 et 174); dans le texte de Taos Amrouche, ce sont «quatre hommes [qui] sortent de la maison de la mariée, portant sur leurs épaules un énorme plat de bois plein de couscous, «le plat de la mariée ». En dehors du trousseau de la mariée, ces cadeaux rituels consistent surtout en échange de nourriture, le partage de nourriture permet de sceller une alliance et, plus, il lui confère une sacralité : aheqq tagella d lemleh, " au nom de la nourriture et du sel partagés », est une formule de serment en kabyle. Des cadeaux rituels peuvent aussi être offerts individuellement à des parents de la mariée comme "le veau de l'oncle", noté dans le texte de Taos Amrouche, offert à l'oncle maternel : il s'agit ici d'une trace ténue de l'un des « deux versants de la parenté berbère ", Germaine Tillion signale en effet des traces du "versant maternel » à l'occasion de cadeau(x) rituel(s) chez « les Touaregs, les paysans de l'Aurès et de Petite Kabylie [....] dans le Moyen Atlas [...] en Mauritanie » (Tillion 1973, p. 47). Ces traces du versant maternel de la parenté sont encore vivantes en certaines régions de Kabylie: aux Illoulen Ousammar (village At Sellam), la mariée séjourne quelques jours avant la noce au sanctuaire de Sidi Slimane et le henné lui est appliqué ; elle peut aussi être invitée par la famille de son oncle maternel ; le cadeau offert à l'occasion des noces par l'oncle maternel à sa nièce revêt toujours une grande importance. Jusqu'aux années 1980, le cadeau offert à l'oncle maternel par la famille du marié est non pas un veau mais un mouton aux cornes, si possible, recourbées ${ }^{3}$.

9 Si pour les deux familles alliées, le moment des noces est placé sous le signe de la " compétition d'honneur ", pour le marié et la mariée, ce moment de passage (au même titre que la naissance et la circoncision) perçu comme un moment de grande vulnérabilité, est accompagné de nombreux rites : aussi bien des rites de protection que des rites propitiatoires. Parmi les rites destinés à assurer la protection du jeune couple, on peut noter :

- La visite ou le séjour dans un lieu saint : il s'agit du sanctuaire d'un marabout (Laoust 1993, p. 73, 93, 99, 168, 169) ou d'un lieu investi d'une sacralité antéislamique (arbre, rocher, source) dénommé azessas, "gardien », en kabyle (Laoust-Chantréaux 1990, p. 198 ; Laoust 1993, p. 168).

- La retraite nuptiale : cette retraite d'une durée de trois ou sept jours est partout observée par la mariée, elle prend fin avec la cérémonie de la ceinture. Cette retraite peut aussi être observée par le marié (Laoust 1993, p. 61, 74, 166); même lorsqu'ils ne sont pas « comme retranchés du monde extérieur » (Laoust 1993, p. 166), cette retraite impose au marié et à la mariée de nombreux interdits destinés à les préserver de tout danger; elle leur impose dans tous les cas une attitude d'extrême réserve : parler peu, manger peu...

- L'imposition du henné : il s'agit d'un moment essentiel dans les cérémonies de mariage, au henné sont attribués non seulement des pouvoirs de protection mais aussi de fécondité 
(Abrous 1992, p. 147). Le lien entre henné et fécondité est tellement étroit que le reste du henné de mariage est très soigneusement conservé ; «si une première naissance tardait, la qibla remettrait à la jeune femme le henné du mariage avec les mêmes rites » (LaoustChantréaux 1990, p. 195). propitiatoires; l'aspect propitiatoire est nettement perceptible dans les rites d'agrégation. Parmi ces très nombreux rites, on en relèvera deux qui sont aussi essentiels que celui de l'imposition du henné : le rite de la ceinture et celui de la coiffe.

- La ceinture : en règle générale, la mariée ne porte pas de ceinture, elle a les cheveux dénoués : «[...] de sept jours [elle] ne doit pas mettre sa longue ceinture de laine (tisfifin), accomplissant par là un rite de magie sympathique partout noté en Berbérie » (LaoustChantréaux 1990, p. 196). Elément essentiel du vêtement féminin, la ceinture est étroitement associée à la maternité (Laoust-Chantréaux 1990, p. 142, 247). La remise de la ceinture après la retraite, de trois ou sept jours, notée dans les textes collectés par Emile Laoust est accompagnée de rites de fécondité (Laoust 1993, p. 82, 100, 114, 119) et (Laoust-Chantréaux 1990, p. 201).

- La coiffe. Le rite de la coiffe, moins perceptible en Kabylie, est signalé dans les textes collectés par Emile Laoust (1993, p. 97, 100, 114, 119, 168, 170). Ce rite, comme celui de la ceinture, parachève la période des noces. La coiffe des femmes mariées étant différente de celle des jeunes filles, ce rite consacre, pour la mariée, un changement de statut; il consiste quelques fois dans le fait de couper « la mèche frontale taunza » de la mariée (Laoust, 1993, p. 97, 100) ; il est également noté pour la Kabylie (Laoust-Chantréaux 1990, p. 201). vêtement féminin, les rites d'accueil de la mariée ont pour siège des endroits de la maison connus pour leur forte charge symbolique: le seuil ou le linteau, la poutre faîtière et le foyer.

- Le seuil est un lieu sur le quel se déroulent aussi bien les rites de séparation au moment du départ de la mariée (Laoust-Chantréaux 1990, p. 197) que les rites d'accueil (Laoust-Chantréaux 1990, p. 198, 199) et Laoust (1993, p. 113, 124, 144). Le seuil étant le lieu de nombreux interdits (Laoust-Chantréaux 1990, p. 39-40), la mariée doit être portée lorsqu'elle le traverse; sur le seuil ont lieu des rites destinés à appeler l'abondance et la fécondité, tels que celui de jeter des dattes, noix, beignets..., d'écraser une grenade, de briser un œuf, etc.

D'autres rites d'accueil - il s'agit surtout de rites propitiatoires - ont pour lieu « le mur qui fait face à la porte d'entrée, le pilier central » (Laoust-Chantréaux 1990, p. 199), la poutre faîtière (Laoust 1993, p. 74, 81), le foyer (Laoust 1993, p. 81, 116). Ce sont des rites d'aspersion ou d'onction utilisant des produits considérés comme bienfaisants (eau, beurre, lait) ; le bris d'un œuf sur la porte ou sur le linteau est lié à la fécondité.

Dans tous ces rites propitiatoires, qui sont très nombreux, la fécondité de la femme est étroitement associée à la fertilité de la terre; ce lien est nettement perceptible à travers les rites qui accompagnent le triage du blé, devant servir au repas de noce ; ce moment, qui est une cérémonie inaugurale, est signalé dans le texte de Taos Amrouche, il l'est aussi par G. Laoust-Chantréaux. Sur le grain déposé en tas est allumée une lampe et sont posés «des noix, des dattes et cinq ou sept œufs. » (Laoust-Chantréaux 1990, p. 192) ; peuvent aussi y être jetées des amandes (Laoust 1993, p. 116) ou des grenades "deux grenades attachées au même rameau » (Laoust 1993, p. 124). La présence de ces fruits à nombreux grains ainsi que des œufs, durant toute les cérémonies du mariage

Encyclopédie berbère, $30 \mid 2010$ 
symbolise l'abondance et la fécondité ; ces mêmes fruits accompagnent les rites des premiers labours d'automne (Laoust-Chantréaux 1990, p. 198).

Dans les textes collectés par E. Laoust, le triage du grain donne lieu à un rituel plus complexe et plus riche que celui observé en Kabylie. Pour cette cérémonie, chez les Inteketto, par exemple, on peut solliciter la présence des timzwar (Laoust 1993, p. 116).

Les combats nuptiaux sont un autre indice à travers lequel on peut lire la dimension propitiatoire des cérémonies de mariage. Ces combats sont signalés dans la quasitotalité des textes collectés par E. Laoust (1993, p. 81, 82, 85, 96, 101, 102...); il s'agit de combats rituels, de simulations car, précise un des textes, «A ce jeu, personne ne se fâche, même si l'on sort de la bagarre quelque peu blessé ». (Laoust 1993, p. 82). Ces combats peuvent opposer les parents du marié à ceux de la mariée, les adolescents et les jeunes filles, les invités, le marié et la mariée ; lorsque le combat oppose le marié et la mariée, l'enjeu est la prééminence au sein du couple, dans les autres cas, l'enjeu est de la nourriture (exemple : bouillie : taharirt, p. 81, gruau, tarwait, p.121), associée à l'abondance.

En règle générale, sont présents lors de ces cérémonies de mariage tous les éléments fondateurs liés au grain et au cycle agraire : soc de charrue (Laoust 1993, p. 158), moulin à bras (Laoust 1993, p. 144, 151), la peau de mouton (alemsir) sur laquelle est posé le moulin à bras au moment de la mouture (Laoust 1993, p. 141, 171).

Il apparaît donc très clairement que ce moment des noces :

- inscrit le mariage dans le système de valeurs avec toutes les pratiques placées sous le signe de la « compétition d'honneur";

- l'enracine dans le système mythico-rituel par ce déploiement de rites ;

- lui confère une sacralité par les visites rendues aux lieux investis de sacralité. La cérémonie religieuse, c'est-à-dire la récitation de la Fatiha et les bénédictions qui l'accompagnent, a lieu au moment des fiançailles ou quelques jours avant la célébration du mariage. C'est cette cérémonie qui rend le mariage licite.

19 La comparaison entre les données relatives à la Kabylie, au Moyen-Atlas et au HautAtlas révèle une profonde similitude dans :

- Le lexique de base : tameyra, "noce"; isli / tislit, "marié / mariée »; tawenza, "mèche frontale, front», associée symboliquement aux notions de chance ou de malchance, considérée comme le lieu où est inscrit le destin ; azenzi (Laoust 1993, p. 95), « chant rituel à l'adresse des fiancées ».

- Le déroulement de la « compétition d'honneur ».

- Les moments essentiels qui jalonnent ces cérémonies de mariage; dans la référence aux mêmes symboles pour les éléments du vêtement (ceinture, coiffe), les parties de la maison [seuil, poutre faîtière, foyer).

- La relation étroite qui lie tout ce rituel au cycle agraire.

Le Moyen-Atlas et le Haut-Atlas se distinguent de la Kabylie par :

- L'existence de noces collectives (Laoust 1993, p. 87 et suivantes);

- Certains rites tels que : lhanna mez̧zin (Laoust 1993, p. 106) [le petit henné] ; lhanna meqqurn (Laoust 1993, p. 107) [grand henné] appelé aussi iḍ n usexsi (Laoust 1993, p. 107) ou « nuit de l'extinction ».

- La récurrence de rites liés avec présence de sandales et babouches, que l'on retrouve aussi chez les Toauregs (cf. Gast 1978). 
Ce rituel lié aux cérémonies de mariage est, aujourd'hui, largement érodé (Abrous 1992); en Kabylie, seuls survivent : la cérémonie religieuse, l'imposition du henné et les chants d'éloge qui, quelques fois, l'accompagnent; quant aux dépenses somptuaires, dernière trace des " compétitions d'honneur ", elles sont, aujourd'hui, rigoureusement réglementées par les assemblées de village ou de tribu. (Abrous 2004, p. 4031, 4032).

\section{BIBLIOGRAPHIE}

ABRous D., 1992 - « Les joutes poétiques du henné : compétition d'honneur et rapt symbolique », Etudes et Documents Berbères, 9, p, 147-164.

ABROUS, D., 2004 - « Kabylie : anthropologie sociale », Encyclopédie berbère XXVI, p 4027-4033. BOURDIEU, P. 1972 - «Le sens de l'honneur chez les Kabyles », Esquisse d'une théorie de la pratique, précédée de trois études d'ethnologie kabyle, Genève, Droz, Genève, p. 15-43.

CHAKER, S., 1995 - « Du pillage et du mariage, des femmes et du bétail. Un champ lexicosémantique curieux en berbère ", Linguistique berbère : études de syntaxe et de diachronie, Paris/ Louvain, Peeters, chap. 17, p. 247-257.

CLAUDOT-HAWAD, H. et HAWAD, 1993 - “Coups et contre-coups : l'honneur en jeu chez les Touaregs ", Les Touaregs portait en fragments, Aix en Provence, Edisud, p. 13-27.

GAST, M., 1978 - « Le don des sandales dans la cérémonie du mariage chez les kel Ahaggar », Actes du $1^{\text {er }}$ congrès d'étude des cultures méditerranéennes d'influence arabo-berbère, Alger, SNED, p. 522-527 ; repris dans : Des Huwwara aux Kel-Ahaggar, Alger, CNRPAH, 2008.

LACOSTE-DUJARDIN, C., 2005 - Dictionnaire de la culture berbère en Kabylie, Paris, La Découverte.

Notices : « Mariage », mariée, "noce".

LAOUST, E., 1993 - Noces berbères. Les cérémonies du mariage au Maroc (Edition établie par Claude Lefébure), Aix-en-Provence, Edisud..

LaOUST-CHANTREAuX, G., 1990 - Kabylie côté femmes. La vie féminine à Aït-Hichem 1937-1939, Aix-enProvence, Edisud.

TILLION, G., 1973 - «Les deux versants de la parenté berbère », Actes du $1^{\text {er }}$ congrès d'étude des cultures méditerranéennes d'influence arabo-berbère, Alger, SNED, p. 43-49.

\section{NOTES}

1. NDLR: On notera que ce terme, construit sur le schème nominal $\operatorname{taC}^{1} C^{2} C^{3} a$, qui est normalement un nom d'action verbal abstrait, repose sur la racine $M \gamma R$, "être grand, important, âgé... ». Tame $r$ ra réfère donc à la " grandeur ", à « l'importance » de la famille. Il est difficile de le relier, comme certains chercheurs l'ont proposé, à la racine $\gamma R$, " appeler " car toute sa morphologie (pluriel, état d'annexion...) est celle d'un nom de schème taccca, qui renvoie nécessairement à une racine triconsonantique $C^{1} C^{2} C^{3}$. Malgré la présence $\mathrm{du} / \mathrm{m} /$ initial, tame $r$ ra est une formation 
strictement parallèle à takerza, tamegra, taferka, tanekra, etc.; le /m/ initial n'a pas lieu ici d'être considéré comme un morphème dérivationnel.

2. NDLR: Sur cette question du lien entre « mariage " et " enlèvement ", on se reportera à l'étude lexico-sémantique de Chaker (1995) qui montre que cette relation n'était peut-être pas, en des

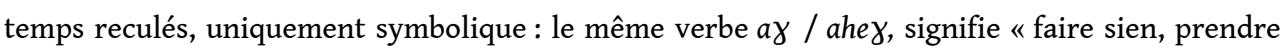
par violence, piller» et «épouser »...Du reste, dans de nombreuses sociétés, africaines notamment, le jeune homme devait réellement enlever sa fiancée et vivre avec elle en brousse pendant plusieurs jours pour prouver qu'il était digne d'elle.

3. Source: information orale communiquée par Saliha Iggui, enseignante au Département da Langue et de Culture Amazigh de l'Université de Béjaia.

4. Timzwar (fem. pluriel de : amzwar : (fem : tamzwart voir Laoust 1993, p. 188, 189, note $\mathrm{n}^{\circ} 1$; pour amezwar, voir Encyclopédie Berbère IV, 1987.

INDEX

Mots-clés : Chant, Ethnologie, Ethnographie, Mariage, Rite, Rituel 